\title{
Repository of SMAD4 Mutations: Reference for Genotype/ Phenotype Correlation
}

\author{
Whitney L. Wooderchak ${ }^{1}$, Zacary Spencer ${ }^{1}$, David K. Crockett ${ }^{1}$, Jamie McDonald ${ }^{1,2}$ and Pınar Bayrak-Toydemir ${ }^{1,3 *}$ \\ ${ }^{1}$ ARUP Institute for Clinical and Experimental Pathology, Salt Lake City, Utah \\ ${ }^{2}$ Department of Radiology, University of Utah, Salt Lake City, Utah \\ ${ }^{3}$ Department of Pathology, University of Utah, Salt Lake City, Utah
}

\begin{abstract}
Mutations in the SMAD4 gene have been found in patients with Juvenile Polyposis Syndrome (JPS), patients with combined Juvenile Polyposis/ Hereditary Hemorrhagic Telangiectasia (JP/HHT) or patients with HHT, but without reported symptoms of juvenile polyposis. We have developed a publicly available, searchable SMAD4 database to aid in genotype/phenotype correlations, using the Human Genome Variation Society recommendations for mutation nomenclature and database content. The purpose of this database is to serve as a repository for all known SMAD4 mutations, variants of uncertain significance, or polymorphisms including sequence based changes and large deletion/ duplications that have been linked to JPS, JP/HHT, or HHT as well as any clinical information or significant literature related to the disorder. Each database entry lists the SMAD4 mutation's location, mutation type, pathogenicity classification, disease-specific phenotype, literature references, and comments (which may include clinical features, complex genotypes, and links to additional literature references). The majority of the SMAD4 database entries consist of missense sequence changes and small deletions with 67 JPS mutations, 23 JP/HHT mutations, and one HHT-causing mutation. Novel SMAD4 variants and updated phenotypic information for any existing database entry can be submitted electronically on the database website (www.arup.utah.edu/database/SMAD4/SMAD4 welcome.php). In conclusion, with links to detailed clinical descriptions and all published references, this database hopes to serve as a valuable reference for the clinical management of patients with JPS, JP/HHT and HHT.
\end{abstract}

Keywords: SMAD4; Juvenile polyposis syndrome; JPS; Hereditary hemorrhagic telangiectasia; HHT; Mutation database; Genotype; Phenotype

Abbreviations: AVMs: Arteriovenous Malformations; BMPR1A: Bone Morphogenic Protein Receptor-1A; HHT: Hereditary Hemorrhagic Telangiectasia; JP/HHT: Juvenile Polyposis/ Hereditary Hemorrhagic Telangiectasia; JPS: Juvenile Polyposis Syndrome; SMAD4/MADH4: Mothers Against Decapentaplegic Homolog 4; TGF- $\beta$ : Transforming Growth Factor-Beta

\section{Introduction}

Juvenile polyposis syndrome (JPS), hereditary hemorrhagic telangiectasia (HHT), JP/HHT and the SMAD4 gene

Juvenile polyposis syndrome (JPS; MIM 174900) is an autosomal dominant disorder that affects 1 in 100,000 individuals [1]. JPS is characterized by the presence of hamartomatous polyps throughout the gastrointestinal (GI) tract and is associated with an increased risk for GI cancers $[2,3]$. Affected individuals may experience abdominal pain, diarrhea and rectal bleeding which can lead to anemia. Juvenile polyposis syndrome is diagnosed when an individual has any one of the following: (1) more than five juvenile polyps of the colon or rectum, (2) multiple juvenile polyps in the upper and lower gastrointestinal tract, or (3) any number of juvenile polyps and a family history of juvenile polyposis syndrome.

JPS is caused by mutations in the gene encoding the mothers against decapentaplegic homolog 4 (SMAD4/MADH4) on chromosome $18 \mathrm{q} 21.1$ or in the gene encoding the bone morphogenic protein receptor-1A (BMPR1A) on chromosome 10q22.3 [4,5]. SMAD4, a tumor suppressor gene and $B M P R 1 A$ are intracellular mediators of the transforming growth factor-beta (TGF- $\beta$ ) signaling pathway [6]. SMAD4 and BMPR1A mutations cause JPS in approximately twenty percent of patients each $[7,8]$. Currently, it is unknown how juvenile polyps form as a consequence of germline SMAD4 or BMPR1A mutations or which additional unidentified genes are responsible for causing the other $60 \%$ of JPS.
Clinical heterogeneity has been observed as a result of SMAD4 mutations. A small number of patients (2-3\%, based on small numbers) clinically diagnosed with Hereditary Hemorrhagic Telangiectasia (HHT; MIM 187300), but without reported symptoms of JPS, have a SMAD4 mutation $[9,10]$. HHT is an autosomal dominant disorder that has distinct, nonoverlapping clinical features from juvenile polyposis. Unlike JPS, HHT is a vascular malformation disorder characterized by recurrent nosebleeds, telangiectases (of the mouth, face, hands, gastrointestinal tract) and arteriovenous malformations (AVMs) in the lung, brain, liver, or spine [11]. The majority of HHT patients $(85 \%)$ have pathogenic mutations in ENG or ACVRL1. These genes, like $S M A D 4$ and BMPR1A, encode proteins involved in the TGF- $\beta$ signaling pathway.

SMAD4 mutations have also been identified in patients from unrelated families meeting the diagnostic criteria for both juvenile polyposis and HHT $[12,13]$. This combined syndrome of juvenile polyposis and HHT (JP/HHT; MIM 175050) is characterized by symptoms of both diseases in different family members in the same family or as the presence of both JPS and HHT in an affected individual $[12,13]$. One previous study found that $22 \%$ of individuals with a SMAD4 mutation in their JPS population also had HHT [14]. But this percentage is likely higher since JPS patients have not historically

*Corresponding author: Pınar Bayrak-Toydemir, M.D., Ph.D., Department of Pathology University of Utah, ARUP Laboratories, 500 Chipeta Way, Salt Lake City, UT 84108, Tel : 801-583-2787 x13171; Fax : 801-584-5207; E-mail: pinar.bayraktoydemir@aruplab.com

Received July 28, 2010; Accepted September 02, 2010; Published September 02, 2010

Citation: Wooderchak WL, Spencer Z, Crockett DK, McDonald J, Bayrak-Toydemir P (2010) Repository of SMAD4 Mutations: Reference for Genotype/Phenotype Correlation. J Data Mining in Genom Proteomics 1:101 doi:10.4172/2153 0602.1000101

Copyright: (C) 2010 Wooderchak WL, et al. This is an open-access article distributed under the terms of the Creative Commons Attribution License, which permits unrestricted use, distribution, and reproduction in any medium, provided the original author and source are credited. 
been well evaluated for HHT manifestations and visa versa. JP/HHT patients seem to have a high frequency of pulmonary AVMs and digital clubbing secondary to hypoxia. The severity (pulmonary, cerebral and hepatic AVMs) and often early onset of HHT symptoms in these patients argue in favor of systemic screening for visceral AVMs in juvenile polyposis patients with SMAD4 mutations [12,13]. But better information about the phenotypic expression of SMAD4 mutations will become available as patients are more fully evaluated for manifestations of both JP and HHT and as mutation positive family members of probands are studied.

\section{Clinical management of JPS}

DNA-based testing for SMAD4 mutations is used to identify individuals at-risk for JPS as well as to determine appropriate medical management. Because SMAD4 mutations can also cause HHT, DNAbased testing for SMAD4 mutations is also used to identify individuals with HHT who do not have an identifiable mutation in ENG or ACVRL1 [9]. If a SMAD4 mutation is identified, the patient should also then be screened for gastric and colonic polyposis [9]. Young individuals with HHT who have GI bleeding or anemia that cannot be attributed to epistaxis or bleeding from telangiectasias should also be evaluated for polyposis.

While most juvenile polyps are benign, malignant transformation can occur. Approximately $20 \%$ of JPS patients have hamartomatous gastrointestinal polyps that turn into malignant lesions [3]. The incidence of colorectal cancer in patients with JPS is also increased ranging from $17-22 \%$ by age 35 years to $68 \%$ by age 60 years. Careful screening of young at-risk individuals and the removal of polyps reduces the malignant potential of this disease.

\section{SMAD4 database objectives}

A database cataloging JPS, JP/HHT, or HHT-causative SMAD4 mutations can be a valuable reference for medical management of patients with these disorders. Drawing SMAD4 genotype/phenotype correlations has been difficult due to drastic variance seen in the number of polyps and the onset of polyp development among members of families with JPS that have the same SMAD4 mutation. Despite this, several genotype/phenotype correlations have been made among individuals with JPS caused by SMAD4 versus BMPR1A mutations. Patients with JPS caused by a $S M A D 4$ mutation are more likely to have upper-GI polyps compared to those with $B M P R 1 A$ or no known mutations and individuals with either a SMAD4 or BMPR1A mutation are more likely than those without mutations identified in these genes to have a more severe phenotype (more than ten lower GI polyps and a family history of GI cancer) $[8,15,16]$.

To aid in the genotype/phenotype correlations that can be drawn from SMAD4 mutations, we developed a publicly available, userfriendly SMAD4 database to document all known SMAD4 mutations or gene variants that have been linked to JPS, HHT, or JP/HHT as well as any clinical information or significant literature related to the disorders. The SMAD4 database is a useful reference for SMAD4 genotype/phenotype correlations due to the availability of phenotype and pathogenicity classifications, descriptive summaries of family clinical information and direct links to each literature reference sited. Because SMAD4 genotype/phenotype correlations are difficult to make for these disorders, it is essential to have a database that provides detailed clinical information, so that in the future, additional genotype/phenotype correlations can be drawn. The database is also linked to all published papers related to each mutation. This feature is different from most databases in that it enables clinicians to easily understand the frequency and detailed clinical scenarios associated with each mutation. Thus, the SMAD4 database is a valuable reference for the medical management of patients with JPS, HHT or JP/HHT.

The database will remain comprehensive and current for JPS, HHT or JP/HHT SMAD4 mutations, with quarterly updates from the literature, clinical laboratory test results and any electronic database submissions. Another useful feature of our database is the electronic data submission, which allows clinical diagnostic laboratories to submit mutations, variants, or polymorphisms without a publication requirement. This feature is extremely valuable because clinical diagnostic laboratories generate large quantities of data but rarely publish these results. This feature is available through disease (or gene) focused databases but not through comprehensive databases. Updates received from colleagues describing novel SMAD4 variants or phenotypic information for any existing database entry will also be updated quarterly. This database serves as the most comprehensive repository for SMAD4 mutations and their associated clinical phenotypes.

\section{Methods}

\section{Data sources}

The SMAD4 database was constructed using SMAD4 mutations obtained from articles found using PubMed (www.ncbi.nlm.nih. gov/sites/entrez) and Google (www.google.com). SMAD4 mutations identified during routine clinical testing performed at ARUP for JPS, $\mathrm{JP} / \mathrm{HHT}$ and HHT were also included in the database. The database was constructed using the Human Genome Variation Society and HUGO's Mutation Database Initiative recommendations for database content (www.hgvs.org/mdifaq.html). The Genbank reference sequences used for the database were NC_000018.9 (genomic DNA), NM_005359 (mRNA, position 1 is "A" of the ATG start codon) and NP_005350.1 (protein).

\section{Software}

The SMAD4 database was developed using PHP and MySQL (MySQL, Inc., Seattle, WA). Coding was performed in-house for dynamic HTML display and JPS SQL tables. Webpages are hosted on a Mac OSX Apache server. All SMAD4 mutations were added to the database and edited using phpMyAdmin.

\section{Results and Discussion}

\section{SMAD4 database website}

The SMAD4 database can be found under Disease Databases on the ARUP Online Scientific Resource webpage (www.arup.utah.edu) or can be accessed directly at www.arup.utah.edu/database/SMAD4/ SMAD4_welcome.php. The SMAD4 database has six webpages (including the SMAD4 Home, Links, Display Database, Search Database, Submissions \& Updates and Contact Us pages) that can easily be navigated using the tabs (Figure 1A). The SMAD4 homepage briefly describes JPS, HHT, JP/HHT and the database aims. In addition, links to the SMAD4 reference sequences are found on the homepage. The SMAD4 Links webpage has links to existing online resources for JPS, HHT, JP/HHT and SMAD4, including the Human Gene Mutation Database (HGMD), MutDB, Uniprot and the Human Protein Reference Database (HPRD). Links to the SMAD4 database exist or are being requested from NCBI, HGMD, HGVS, UniProt, GENATLAS, GENETests and The HHT Foundation websites. These links will integrate the SMAD4 database into relevant scientific fronts. 


\section{ARDP}

\section{ARUP ONLINE SCIENTIFIC RESOURCE}

Home Disease Databases $\boldsymbol{\nabla}$ Educational Resources $\mathbf{v}$ Collaborate With Us $\boldsymbol{\nabla}$ Visiting ARUP $\mathbf{v}$ U of U Pathology

A

\begin{tabular}{llllll}
\hline SMAD4 Home & SMAD4 Links & Display Database & Search Database & Database Submissions Contact Us
\end{tabular}

\section{SMAD4 Database Display Page}

- Click the arrows to sort by Mutation Type, Classification, or Reference columns. Place mouse over icon for Comments.

- For cDNA numbering, nucleotide 1 is the "A" of the ATG start codon.

- Frame Shifts are documented by stating the original amino acid followed by the codon number and word frameshift.

- Disease abbreviations are Juvenile Polyposis Syndrome (JPS), Hereditary Hemorrhagic Telangiectasia (HHT), and the combined syndrome (JP/HHT).

86 records found.

B

\begin{tabular}{|c|c|c|c|}
\hline Location & $\stackrel{\triangle}{\text { Mutation Type }}$ & $\begin{array}{l}\text { Nycleotide } \\
\text { Change }\end{array}$ & $\begin{array}{l}\text { Proteiin } \\
\text { Change }\end{array}$ \\
\hline Intron 02 & Splice Site & c. $1-128+1 G>A$ & \\
\hline Exon 03 & Deletion, Insertion & c.189_197dellins44 & p.Thr63 frameshift \\
\hline Exon 06 & Nonsense & c. $538 \mathrm{C}>\mathrm{T}$ & p.GIn180X \\
\hline Exon 06 & Deletion & c.516_527del & p.Leu172 frameshift \\
\hline Exon 12 & Nonsense & c. $1333 \mathrm{C}>\mathrm{T}$ & p.Arg445X \\
\hline Exon 13 & Deletion & c.1564_1565delCC & p.Pro522 frameshift \\
\hline Exon 10 & Missense & c. $970 \mathrm{~T}>\mathrm{C}$ & p.Cys32 Arg \\
\hline Exon 10 & Deletion & c.971delG & p.Cys324 frameshift \\
\hline Exon 11 & Nonsense & c. $1193 \mathrm{G}>\mathrm{A}$ & p.Trp398X \\
\hline Exon 12 & Deletion & c.1411-1435del25 & p.Gly471 frameshift \\
\hline Exon 13 & Duplication & c.1586dupA & p.Leu529 frameshift \\
\hline Intron 08 & & c. $905-52 A>G$ & \\
\hline
\end{tabular}

$\begin{array}{lcc}\begin{array}{c}\text { Classification } \\ \text { Mutation }\end{array} & \text { JP } & \underline{\text { Woodford-Richens (2001) }} \\ \text { Mutation } & \text { JP } & \underline{\text { Woodford-Richens (2001) }} \\ \text { Mutation } & \text { JP } & \underline{\text { Woodford-Richens (2001) }} \\ \text { Mutation } & \text { JP } & \underline{\text { Woodford-Richens (2001) }} \\ \text { Mutation } & \text { JP } & \underline{\text { Woodford-Richens (2001) }} \\ \text { Mut Male patient, > 100 gastric juvenile polyps, 8 } \\ \text { Mut colorectal juvenile polyps } \\ \text { Mutation } & \text { JP } & \underline{\text { van Hattem (2008) }} \\ \text { Mutation } & \text { JP } & \underline{\text { van Hattem (2008) }} \\ \text { Mutation } & \text { JP } & \underline{\text { van Hattem (2008) }} \\ \text { Mutation } & \text { JP } & \underline{\text { van Hattem (2008) }} \\ \text { Polymorphism } & \text { JP } & \underline{\text { Unpublished ARUP data }}\end{array}$

Figure 1: The SMAD4 database display. (A) Tabs facilitate navigation within the database website. (B) Database column headings. Entries can be sorted alphabetically by clicking the sort arrows within the Classification or Phenotype column headings in ascending $(\boldsymbol{\Delta})$ or descending ( $\boldsymbol{\nabla})$ order. (C) Example of information listed in the comments, including relevant clinical information.

The SMAD4 database is found on the Display Database webpage shown in Figure 1. As of the initial public release date, January 2010, the database contained 77 entries. The database currently contains 86 entries after the first update in April 2010. The default database display is sorted by the location of the SMAD4 sequence variation (5' to 3') and all sequence variation is heterozygous unless otherwise noted. Figure 1B shows the database display columns (Location, Mutation Type, Nucleotide Change, Protein Change, Classification, Phenotype, References and Comments described further below). Comments for each entry may provide specific clinical or biochemical information related to the mutation described. Phenotype abbreviations are defined above the database column headings. A search can be initiated from the SMAD4 Home or Search Database pages.

In order to enable researchers to submit novel SMAD4 sequence variation or update information for existing entries, a Database Submissions webpage was generated. A Contact Us webpage displays current email addresses for the database curators.

\section{SMAD4 database display columns}

SMAD4 Mutation location and mutation type: The Location column (Figure 1B) displays the exon or intron number for each SMAD4 sequence variation. Figure $2 \mathrm{~A}$ depicts the mutation type distribution for patients with JPS, JP/HHT and HHT. The majority of the database entries are missense sequence changes and small deletions. A smaller number of entries describe nonsense mutations, duplications, deletion/insertions, splice site alterations, or large deletions (Figure 2A).

Of the 86 SMAD4 database entries, 67 SMAD4 mutations caused juvenile polyposis ( 19 missense mutations, 19 deletions, 10 nonsense mutations, 7 insertions, 4 duplications, 4 splice site variants, 3 large deletions including 1 deletion of the entire gene and 1 insertion/ deletion). JPS-causing mutations were identified in all thirteen exons with the majority (70\%) occurring in SMAD4 exons 10-13 (Figure $2 \mathrm{~B})$. Of the $47 \mathrm{JPS}$-causing mutations identified in this region, exons 10 and 13 had the highest number of mutations $(27 \%$ and $21 \%$, respectively) while exons 11 and 12 had the remaining $22 \%$ of JPS 


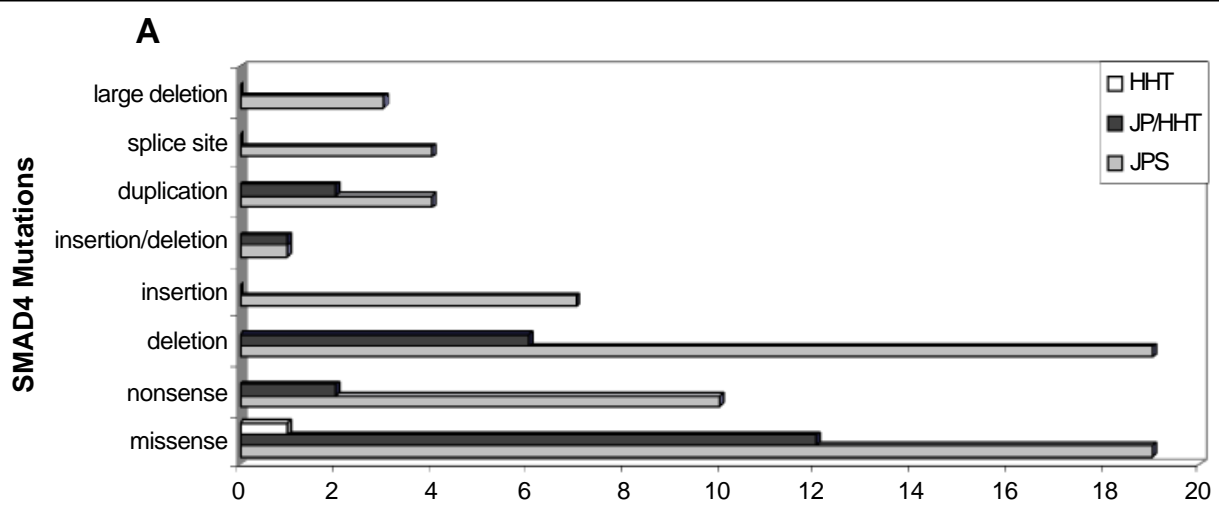

B

SMAD4 Database Entries

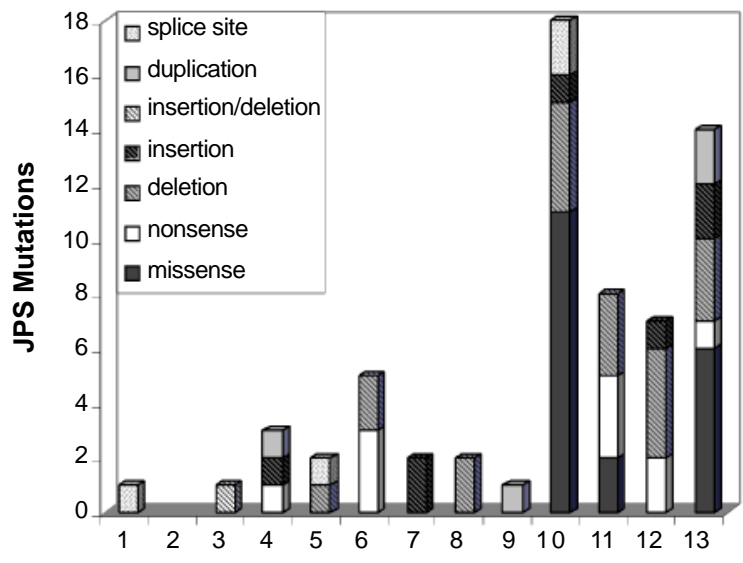

C

SMAD4 exon

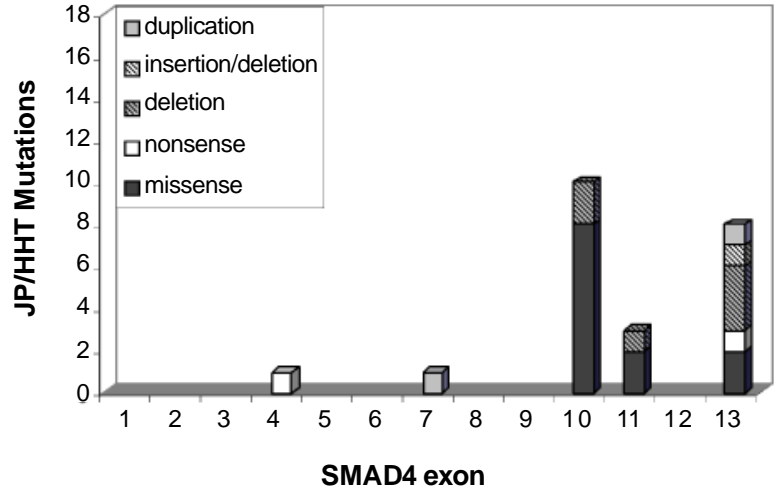

Figure 2: SMAD4 mutations in JPS, JP/HHT and HHT patients. (A) Distribution of SMAD4 mutation database entries categorized by mutation type and phenotype (JPS in gray, JP/HHT in black and HHT in white). (B) Number of different JPS-causing SMAD4 mutations in each exon of the SMAD4 gene. Mutations in each exon were further classified by mutation type (see inset). Three large SMAD4 deletions, one of the entire gene and two others beginning with exon 7 and exon 8 , are not shown. (C) Number of different JP/HHT-causing SMAD4 mutations in each exon of the SMAD4 gene. Mutations in each exon were further classified by mutation type (see inset).

mutations. The most common SMAD4 mutation, a deletion in exon 11 (c.1244_1247delACAG, p.Asp415 frameshift), has been reported in multiple unrelated families with JPS $[3,4,8,16-22$, unpublished ARUP results]. Exons 10-13 code for the SMAD4 C-terminal MH2 domain which plays an important role in SMAD4 oligomerization and transcriptional activation $[23,24]$.

Most aberrations that caused JPS in this region were missense mutations and small deletions (Figure 2B). The majority of missense mutations caused SMAD4 protein charge alterations in exons 10
(Arg361Ser/Gly/Cys/His, Glu330Lys/Gly, Gly352Arg, Cys324Arg and Cys363Arg), 11 (Gly386Asp and Glu390Lys) and 13 (Asp493Ala and Trp509Arg) [7,8,14,15,20,22,25-30, unpublished ARUP data]. The remaining missense mutations from exons 10 and 13 altered the size rather than the charge of the amino acid from the SMAD4 MH2 domain (Tyr353Ser, Leu364Trp, Gly491Val, Gly510Val, Trp524Leu and Leu533Val) $[3,7,14,18,20,22,29]$. Mutations predicted to lead to a frameshift and eventually cause the formation of a premature stop codon were found in exon 10 (4 deletions and 1 insertion), exon 11 ( 3 deletions), exon 12 (4 deletions and 1 insertion) and 
exon 13 (3 deletions, 2 insertions and 2 duplications) $[3,4,7,8,14,16$ $22,26,28,30$, unpublished ARUP results]. Six nonsense mutations that also caused a premature stop codon to form were identified in exons 11 (c.1162C>T, p.Glu388X; c.1193G>A, p.Trp398X; c.1236C >G, p.Tyr412X), 12 (c.1333C >T, p.Arg445X; c.1342C >T, p.Gln448X) and 13 (c.1527G>A, p.Trp509X) [3,7,8,14,16,21,22,26-28,31]. Two mutations predicted to alter splicing in this region were found in intron 10 (c.1139G $>$ A and c.1139+1G >A) [14,20,22].

The remaining $30 \%$ of JPS SMAD4 mutations were located in exons 1-9 with the highest frequency in exons 4 and 6 (Figure 2B). Mutations in the SMAD4 N-terminal MH1 DNA binding domain (comprised of exons 2-4) can significantly reduce the DNA binding activity of $S M A D 4$ to its target genes while mutations in the linker region of SMAD4 (comprised of exons 5-9) affect transcription activation of the SMAD complex [32,33]. Most of the JPS SMAD4 mutations in these regions led to a frameshift in the SMAD4 protein or were caused by point mutations leading to a truncated protein. No missense mutations were located in exons 1-9. Mutations predicted to lead to a frameshift leading to a premature stop codon were found in exon 3 (an insertion/deletion), exon 4 (1 duplication and 1 insertion), exon 5 ( 1 deletion), exon 6 ( 2 deletions), exon 7 ( 2 insertions), exon 8 (2 deletions) and exon 9 ( 1 duplication) $[3,4,7,8,14,16,17,20,22,26,34$, 35]. Four nonsense mutations that lead to a premature stop codon were also identified in exon 4 (c.403C $>$ T, p.Arg135X) and exon 6 (c.437T >A, p.Leu146X; c.533C > G, p.Ser178X; c.538C > T, p.Gln180X) $[14,18,20,29,34]$. Two mutations predicted to alter splicing were found in intron 1 (c.1-128+1G>A) and intron 4 (c.425-6A near exon 5) $[14,34]$.

Twenty-three SMAD4 mutations caused JP/HHT (12 missense, 6 deletions, 2 nonsense, 2 duplications and 1 insertion/deletion) and one missense mutation in exon 10 (c.1081C > T, p.Arg361Cys) caused HHT $[9,12,13,20]$. The exon distribution of the JP/HHT SMAD4 mutations is shown in Figure 2C. The distribution of these mutations follows the same trend as what is seen with the JPS patients (Figure 2B). Prior to the identification of twelve additional SMAD4 mutations in 15 additional JP/HHT patients [13], all previously identified JP/HHT SMAD4 mutations were limited to the MH2 domain of the SMAD4 protein (exons 10-13). Although most of the JP/HHT SMAD4 mutations identified in this new cohort of patients clustered in the MH2 domain (exons 10-13), two novel SMAD4 mutations were identified in exons 4 (c.302G $>$ Z, p.W101X) and 7 (c.692dupG, p.S232 frameshift) indicating that JP/HHT SMAD4 mutations are not limited to the MH2 domain [13]. The MH1 domain had one missense mutation in exon 4 , the linker domain had one duplication resulting in a frameshift in exon 7 and the MH2 domain had the most mutations ( 8 missense mutations and 2 small deletions in exon 10,2 missense mutations and 1 small deletion in exon 11 and 2 missense, 1 nonsense, 3 deletions, 1 deletion/insertion and 1 duplication in exon $13[9,12,13,20]$. Contrary to the JPS SMAD4 mutations, no large SMAD4 deletions/duplications or splice site mutations cause JP/HHT (Figure 2A and 2C).

Nucleotide and protein change: Nucleotide and protein changes are listed according to the Human Genome Variation Society recommendations for sequence variation nomenclature. The first cDNA nucleotide (c.1) corresponded to the "A" of the ATG start codon of the SMAD4 Genbank reference sequence (NM_005359.5). For complex SMAD4 sequence variation mutations involving deletions, duplications, or insertion/deletions, the codon number listed is the first codon involved in the change. For splice site mutations and intronic polymorphisms, only the genotype is displayed. Single amino acid changes are listed in the Protein Change column. Frame Shifts are documented by stating the original amino acid followed by the codon number and word frameshift. For large SMAD4 deletions, a description of the deleted exons is provided in the Protein Change column. For more complex mutations, a brief description of the protein changes may appear in the Comments.

Classification of pathogenicity: Any deleterious, germline SMAD4 sequence change that causes JPS, HHT, or JP/HHT and segregates with the JPS disease symptoms within a family is defined as a "mutation". SMAD4 "polymorphisms" are benign, germline SMAD4 sequence changes that are not causative of JPS, HHT, or JP/HHT. The SMAD4 database has 86 mutation entries associated with JPS, JP/HHT or HHT as of the first update (April 2010). Of the 86 germline variants that comprise the SMAD4 database, 83 are classified as mutations and 3 are polymorphisms (c.354G >A, p.Ala118Ala in exon 4; c.788-32A > T in intron 7 and c.905-52A $>G$ in intron 8) [20,30, unpublished ARUP results]. Comments may have additional information to support the classification designation.

Phenotypes caused by SMAD4 mutations: Clinical heterogeneity has been observed as a result of SMAD4 mutations. Brief descriptions of the JPS, HHT and JP/HHT phenotypes caused by SMAD4 mutations are found on the SMAD4 Home Page. Abbreviations for these phenotypes are listed at the top of the Display Database and Search Database pages. Most SMAD4 mutations were unique to either the JPS or JP/HHT phenotype.

When the database was initially launched, the majority of the JPS SMAD4 mutations (63 of 67 entries) had no phenotypic overlap with JP/HHT. Seven mutations were unique to the JP/HHT phenotype: one in exon 10 (c.1088 1090delGTT, p.Cys363del) and six in exon 13 (c.1594delG, p.Ala532 frameshift; c.1596_1597delCCinsT, p.Ala532 frameshift; c.1596delC, p.Leu533 frameshift; c.1598T >G, p.Leu533Arg; c.1600C > T, p.Gln534X and c.1612 1625del, p.Glu538 frameshift) $[9,12,20]$. Including the findings from Gallione and coworkers, 16 of the 23 JP/HHT SMAD4 mutations associated clinically with JP/HHT alone while 60 out of 67 SMAD4 mutations were unique to the JPS phenotype [13].

Seven SMAD4 mutations displayed phenotypic variability with most residing within 12 amino acids of each other on the $\mathrm{MH} 2$ domain. A missense mutation in exon 10 (c.1081C > T, p.Arg361Cys) displayed the most phenotypic variability, as patients with JPS, HHT, or JP/HHT had the same mutation. This is the only SMAD4 mutation identified thus far to strictly cause HHT and not the combined syndrome JP/ HHT [9]. Six additional SMAD4 mutations displayed phenotypic variability where patients with the same mutation exhibited clinical findings consistent with JPS or JP/HHT in exons 10 and 11 (c.988G $>$ A, p.Glu330Lys; c.1054G >A, p.Gly352Arg; c.1081C >G, p.Arg361Gly; c.1082G >A, p.Arg361His; c.1091T>G, p.Leu364Trp; c.1157G >A, p.Gly386Asp) [12-15,20,27,29].

Adding to the complexity of these disorders, JP/HHT-causing SMAD4 mutations were recently shown to not be limited to the MH2 domain [13]. Recently, two JP/HHT mutations were identified in other parts of the gene (exons 4 and 7) indicating that the cumulative SMAD4 mutational spectra for JPS and JP/HHT are almost identical allowing no apparent molecular distinction between JPS and JP/HHT [13]. We anticipate that more information about the phenotypic expression of SMAD4 mutations will become available as patients are more fully evaluated for manifestations of both JP and HHT and as mutation positive family members of probands are studied. As more 
patients with JPS are examined for HHT characteristics, we predict that the genotype/phenotype overlap among JPS and JP/HHT SMAD4 mutations that are currently distinct will increase.

References: The first literature report to publish information on each SMAD4 sequence variation is listed in the Reference column. Each reference is hyperlinked to their corresponding abstract in PubMed. Additional references that report the same variation can be found in the comments. References that used sequence variation nomenclature that was different or obsolete is noted in the comments. For example, the most common JPS-causing SMAD4 mutation (c.1244_1247 delACAG, p.Asp415 frameshift) had many listed references and the comments reflect this.

Comments: The comments for each SMAD4 database entry are visualized by placing the mouse pointer over the Comments column icon as demonstrated in Figure 1C. Comments may further explain information displayed for each database entry and often contain specific clinical information derived from multiple literature references which are distinguished as (author name, year). Links to these references are listed in the References column. Comments may also provide specific biochemical information related to the pathogenicity of the variant described.

\section{Database sort and search functions}

The SMAD4 database can be searched by location, classification, codon number, phenotype, or protein change from the SMAD4 Home or Search Database webpages. The database display can also be sorted alphabetically in ascending or descending order, by clicking the arrows found within the classification, phenotype, or reference column headings (Figure 1B).

\section{Database submissions and updates}

New SMAD4 sequence variation can be submitted to the SMAD4 database using the Database Submissions webpage. This form can also be used to update clinical information or to clarify the current classification or phenotype of SMAD4 sequence variants. The submission form requests information on the sequence variation, clinical consequences, family history, publications and contact information for the submitter. New database submissions and updates will be reviewed and verified by the database curators, who will designate the classification and JPS phenotype based on the clinical information provided.

Quarterly content updates will be performed to maintain the accuracy and utility of the SMAD4 database using new SMAD4 sequence variation information from literature reports, electronic database submissions and updates and routine clinical testing performed at ARUP. The date of the latest update is noted on the SMAD4 Home webpage. By encouraging external data submissions and updating the database on a continual basis, we expect to maintain the SMAD4 database as the most comprehensive, publically available $S M A D 4$ genotype/phenotype data repository.

\section{References}

1. Burt RW, Bishop DT, Lynch HT, Rozen P, Winawer SJ (1990) Risk and surveillance of individuals with heritable factors for colorectal cancer. WHO Collaborating Centre for the Prevention of Colorectal Cancer. Bull World Health Organ 68: 655-665.

2. Jarvinen H, Franssila KO (1984) Familial juvenile polyposis coli; increased risk of colorectal cancer. Gut 25: 792-800.

3. Handra-Luca A, Condroyer C, de Moncuit C, Tepper M, Fléjou JF, et al. (2005) Vessels' morphology in SMAD4 and BMPR1A-related juvenile polyposis. Am J Med Genet A 138A: 113-117.
4. Howe JR, Roth S, Ringold JC, Summers RW, Järvinen HJ, et al. (1998) Mutations in the SMAD4/DPC4 gene in juvenile polyposis. Science 280 : 1086-1088.

5. Howe JR, Bair JL, Sayed MG, Anderson ME, Mitros FA, et al. (2001) Germline mutations of the gene encoding bone morphogenetic protein receptor $1 \mathrm{~A}$ in juvenile polyposis. Nat Genet 28: 184-187.

6. Heldin $\mathrm{CH}$, Miyazono K, ten Dijke P (1997) TGF-beta signaling from cell membrane to nucleus through SMAD proteins. Nature 390: 465-471.

7. Howe JR, Sayed MG, Ahmed AF, Ringold J, Larsen-Haidle J, et al. (2004) The prevalence of MADH4 and BMPR1A mutations in juvenile polyposis and absence of BMPR2, BMPR1B and ACVR1 mutations. J Med Genet 41: 484 491.

8. Sayed MG, Ahmed AF, Ringold JR, Anderson ME, Bair JL, et al. (2002) Germline SMAD4 or BMPR1A mutations and phenotype of juvenile polyposis. Ann Surg Oncol 9: 901-906.

9. Gallione CJ, Richards JA, Letteboer TG, Rushlow D, Prigoda NL, et al. (2006) SMAD4 mutations found in unselected HHT patients. J Med Genet 43: 793797.

10. Prigoda NL, Savas S, Abdalla SA, Piovesan B, Rushlow D, et al. (2006) Hereditary haemorrhagic telangiectasia: mutation detection, test sensitivity and novel mutations. J Med Genet 43: 722-728.

11. Bayrak-Toydemir P, McDonald J, Markewitz B, Lewin S, Miller F, et al. (2006) Genotype-phenotype correlation in hereditary hemorrhagic telangiectasia: mutations and manifestations. Am J Med Genet A 40: 463-470.

12. Gallione CJ, Repetto GM, Legius E, Rustgi AK, Schelley SL, et al. (2004) A combined syndrome of juvenile polyposis and hereditary haemorrhagic telangiectasia associated with mutations in MADH4 (SMAD4). Lancet 363 : 852-859.

13. Gallione C, Aylsworth AS, Beis J, Berk T, Bernhardt B, et al. (2010) Overlapping spectra of SMAD4 mutations in juvenile polyposis (JP) and JPHHT syndrome. Am J Med Genet A 152A: 333-339.

14. Aretz S, Stienen D, Uhlhaas S, Stolte M, Entius MM, et al. (2007) High proportion of large genomic deletions and a genotype phenotype update in 80 unrelated families with juvenile polyposis syndrome. J Med Genet 44: 702 709 .

15. Burger B, Uhlhaas S, Mangold E, Propping P, Friedl W, et al. (2002) Novel de novo mutation of MADH4/SMAD4 in a patient with juvenile polyposis. Am J Med Genet 110: 289-291.

16. Friedl W, Uhlhaas S, Schulmann K, Stolte M, Loff S, et al. (2002) Juvenile polyposis: massive gastric polyposis is more common in $\mathrm{MADH} 4$ mutation carriers than in BMPR1A mutation carriers. Hum Genet 111: 108-111.

17. Friedl W, Kruse R, Uhlhaas S, Stolte M, Schartmann B, et al. (1999) Frequent 4-bp deletion in exon 9 of the SMAD4/MADH4 gene in familial juvenile polyposis patients. Genes Chromosomes Cancer 25: 403-406.

18. Roth S, Sistonen P, Salovaara R, Hemminki A, Loukola A, et al. (1999) SMAD genes in juvenile polyposis. Genes Chromosomes Cancer 26: 54-61.

19. Howe JR, Shellnut J, Wagner B, Ringold JC, Sayed MG, et al. (2002) Common deletion of SMAD4 in juvenile polyposis is a mutational hotspot. Am J Hum Genet 70: 1357-1362.

20. Pyatt RE, Pilarski R, Prior TW (2006) Mutation screening in juvenile polyposis syndrome. J Mol Diagn 8: 84-88.

21. Pintiliciuc OG, Heresbach D, de-Lajarte-Thirouard AS, Dugast $C$, Reignie A, et al. (2008) Gastric involvement in juvenile polyposis associated with germline SMAD4 mutations: an entity characterized by a mixed hypertrophic and polypoid gastropathy. Gastroenterol Clin Biol 32: 445-450.

22. Calva-Cerqueira D, Chinnathambi S, Pechman B, Bair J, Larsen-Haidle J, et al. (2009) The rate of germline mutations and large deletions of SMAD4 and BMPR1A in juvenile polyposis. Clin Genet 75: 79-85.

23. Liu F, Pouponnot C, Massagué J (1997) Dual role of the Smad4/DPC4 tumor suppressor in TGFbeta-inducible transcriptional complexes. Genes Dev 11: 3157-3167.

24. Liu F, Hata A, Baker JC, Doody J, Cárcamo J, et al. (1996) A human Mad protein acting as a BMP-regulated transcriptional activator. Nature 381: 620 623

25. Houlston R, Bevan S, Williams A, Young J, Dunlop M, et al. (1998) Mutations in DPC4 (SMAD4) cause juvenile polyposis syndrome, but only account for a minority of cases. Hum Mol Genet 7: 1907-1912. 
Citation: Wooderchak WL, Spencer Z, Crockett DK, McDonald J, Bayrak-Toydemir P (2010) Repository of SMAD4 Mutations: Reference for Genotype/ Phenotype Correlation. J Data Mining in Genom Proteomics 1:101 doi:10.4172/2153-0602.1000101

Page 7 of 7

26. Woodford-Richens K, Bevan S, Churchman M, Dowling B, Jones D, et al. (2000) Analysis of genetic and phenotypic heterogeneity in juvenile polyposis. Gut 46: 656-660.

27. Kim IJ, Ku JL, Yoon KA, Heo SC, Jeong SY, et al. (2000) Germline mutations of the dpc4 gene in Korean juvenile polyposis patients. Int J Cancer 86: 529532 .

28. van Hattem WA, Brosens LA, de Leng WW, Morsink FH, Lens S, et al. (2008) Large genomic deletions of SMAD4, BMPR1A and PTEN in juvenile polyposis. Gut 57: 623-627.

29. Alazzouzi $H$, Alhopuro $P$, Salovaara $R$, Sammalkorpi $H$, Järvinen $H$, et al. (2005) SMAD4 as a prognostic marker in colorectal cancer. Clin Cancer Res 11: $2606-2611$

30. Sweet K, Willis J, Zhou XP, Gallione C, Sawada T, et al. (2005) Molecular classification of patients with unexplained hamartomatous and hyperplastic polyposis. JAMA 294: 2465-2473.
31. Lamireau T, Olschwang S, Rooryck C, Le Bail B, Chateil JF, et al. (2005) SMAD4 germinal mosaicism in a family with juvenile polyposis and hypertrophic osteoarthropathy. J Pediatr Gastroenterol Nutr 41: 117-120.

32. Jones JB, Kern SE (2000) Functional mapping of the MH1 DNA-binding domain of DPC4/SMAD4. Nucleic Acids Res 28: 2363-2368.

33. de Caestecker MP, Yahata T, Wang D, Parks WT, Huang S, et al. (2000) The Smad4 activation domain (SAD) is a proline-rich, p300-dependent transcriptional activation domain. J Biol Chem 275: 2115-2122.

34. Woodford-Richens KL, Rowan AJ, Poulsom R, Bevan S, Salovaara R, et al (2001) Comprehensive analysis of SMAD4 mutations and protein expression in juvenile polyposis: evidence for a distinct genetic pathway and polyp morphology in SMAD4 mutation carriers. Am J Pathol 159: 1293-1300.

35. Shikata K, Kukita Y, Matsumoto T, Esaki M, Yao T, et al. (2005) Gastric juvenile polyposis associated with germline SMAD4 mutation. Am J Med Genet A 134: 326-329. 